International Journal of Bifurcation and Chaos, Vol. 29, No. 6 (2019) 1930014 (17 pages)

(C) The Author(s)

DOI: $10.1142 /$ S0218127419300143

\title{
Invariant Patterns for Replicator Dynamics on a Hexagonal Lattice
}

\author{
K. Ikeda*, Y. Kogure ${ }^{\dagger}$ and H. Aizawa ${ }^{\ddagger}$ \\ Department of Civil and Environmental Engineering, \\ Tohoku University, Aoba, Sendai 980-8579, Japan \\ *kiyohiro.ikeda.b4@tohoku.ac.jp \\ †yosuke.kogure.t2@dc.tohoku.ac.jp \\ 部吕i.aizawa.p3@dc.tohoku.ac.jp \\ Y. Takayama \\ Institute of Science and Engineering, Kanazawa University, \\ Kakuma, Kanazawa 920-1192, Japan \\ ytakayama@se.kanazawa-u.ac.jp
}

Received November 26, 2018

\begin{abstract}
A hexagonal lattice is a promising and plausible spatial platform for economic agglomeration in spatial economic models. This paper aims at the elucidation of agglomeration mechanisms for the replicator dynamics on this lattice. Attention is paid to the existence of invariant solutions that retain their spatial patterns when the bifurcation parameter changes. Such existence is a special feature of the replicator dynamics, which is widely used in economics. A theoretical procedure to find invariant patterns is proposed and possible invariant patterns are advanced and classified. Among a plethora of theoretically possible invariant patterns, those which actually become stable for a spatial economic model are investigated numerically. The major finding of this paper, is the demonstration of equilibrium curves of invariant patterns that are connected by those of noninvariant ones to form a complicated mesh-like structure, just like the threads of warp and weft. It would be an important scientific mission to elucidate the mechanism of this complicated structure, and contribute to the study in economic geography.
\end{abstract}

Keywords: Bifurcation; group-theoretic bifurcation theory; hexagonal lattice; invariant pattern; replicator dynamics; spatial economic model.

\section{Introduction}

The self-organization of hexagonal distributions of cities, which was envisaged in the central place theory of economic geography Christaller, 1933; Lösch, 1954, has been simulated by the numerical bifurcation analysis of a spatial economic model on a hexagonal lattice Ikeda et al.. 2012b: Ikeda \& Murota, 2014; Ikeda et al., 2018a]. In this analysis, we have observed a plethora of direct and further bifurcating patterns from the uniform state that form a complicated network of equilibrium curves. This paper aims to elucidate the mechanism of this complicated network in the light of geometrical symmetry.

As a hint of this, we refer to an example of bifurcation for the replicator dynamics on a hexagonal

\footnotetext{
*Author for correspondence

This is an Open Access article published by World Scientific Publishing Company. It is distributed under the terms of the Creative Commons Attribution 4.0 (CC-BY) License. Further distribution of this work is permitted, provided the original work is properly cited.
} 


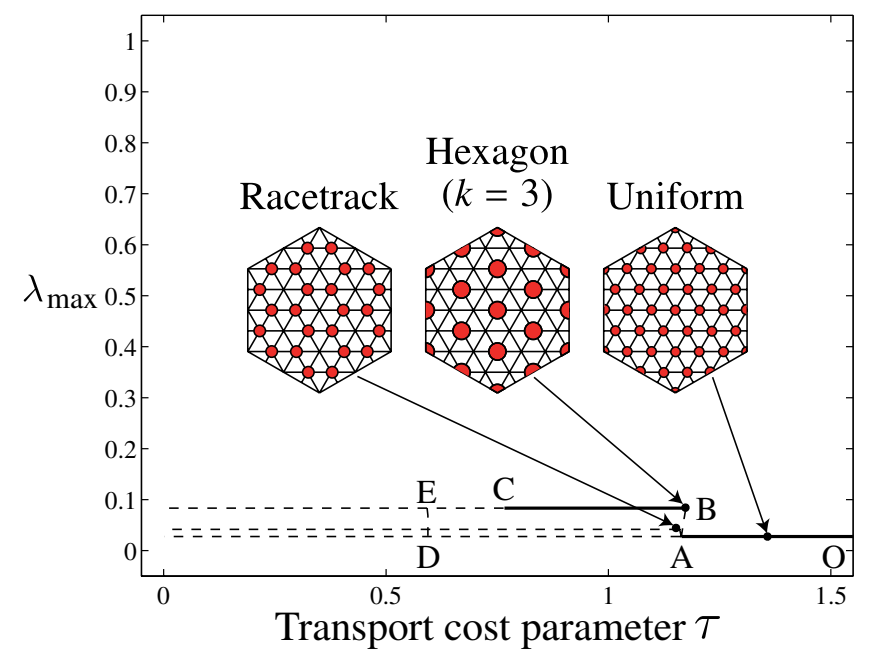

Fig. 1. Bifurcating patterns emerged from the uniform state on a hexagonal lattice [keda et al., 2018a, Fig. 6(a)]. The hexagonal domains denote population distributions for the associated equilibria that are distributed on the nodes of the lattice; the abscissa denotes a bifurcation parameter (transport $\operatorname{cost} \tau$ ); the ordinate expresses the maximum population in the distributed population.

lattice as shown in Fig. 1. From the curve OA of the uniform state, a bifurcating curve $\mathrm{AB}$ branches and arrives at the curve $\mathrm{BC}$ of a hexagonal distribution. It is to be noted that the curves $\mathrm{OA}$ and $\mathrm{BC}$ are horizontal as the population distribution remains constant along these curves when the bifurcation parameter changes. We may wonder why these curves are horizontal! The existence of such constant distributions, called invariant patterns [Ikeda et al., 2018b, 2018c], has been acknowledged in spatial economic models with the replicator dynamics, which is widely used in economics [Sandholm, 2010]. The horizontal curves are connected by nonhorizontal ones, $\mathrm{AB}$ and DE, to form a mesh-like structure. Moreover, we encounter, in this paper (Sec. 5), a complicated mesh-like structure with a large number of horizontal and nonhorizontal curves, just like threads of warp and weft. It would be an important scientific mission to elucidate the mechanism of such a complicated structure and contribute to the study of economic agglomerations.

That said, this paper carries out a theoretical study of invariant patterns for the replicator dynamics on a hexagonal lattice. We consider a scalar field of a finite group $\mathrm{D}_{6} \dot{+}\left(\mathbb{Z}_{n} \times \mathbb{Z}_{n}\right)$ to express the symmetry of an $n \times n$ hexagonal lattice with periodic boundary conditions 11 The mechanism of symmetry-breaking bifurcations, which is a mainstream of nonlinear mathematics Golubitsky et al., 1988], is combined with the mechanism of invariant patterns proposed in this paper. A group-theoretic condition for the existence of invariant patterns is presented and is employed to obtain invariant patterns on a hexagonal lattice exhaustively.

In the numerical bifurcation analysis of a symmetric system, it is customary to obtain the uniform solution and direct, secondary, tertiary bifurcating solutions successively, and, in turn, to find a family of stable solutions. In this paper, in view of the existence of invariant solutions, we propose the following innovative bifurcation analysis procedure to find stable equilibrium curves:

- Obtain all invariant patterns and investigate their stability.

- Obtain bifurcating equilibrium curves connecting stable invariant solutions and investigate their stability.

This procedure is applied to a spatial economic model [Forslid \& Ottaviano, 2003] in economic geography to observe a network structure of equilibrium curves. Such an application could contribute to the study of economic geography, in which economic agglomeration is studied mostly for simple spatial platforms, including: a two-location economy [Fuiita et al., 1999; Baldwin et al., 2003], a long narrow economy on an infinite line or a line segment [Fujita \& Mori, 1997; Mori, 1997], and a racetrack economy on the circumference of a circle Tabuchi \& Thisse, 2011; Ikeda et al., 2012a; Akamatsu et al., 2012]. Invariant patterns in a racetrack economy were also investigated Castro et al., 2012; Ikeda et al., 2012a; Ikeda et al., 2018c.

There are a number of related studies in natural science and social science. Mathematical mechanism of the Bénard problem [Bénard, 1900] was studied using a hexagonal lattice Sattinger, 1978]. Equivariant bifurcation analysis of a $\mathrm{D}_{6} \dot{+} \mathrm{T}^{2}$-symmetric hexagonal lattice has been conducted to search for possible bifurcating patterns Kirchgässner, 1979; Buzano \& Golubitskv, 1983; Dionne \& Golubitsky, 1992; Dionne et al., 1997: Judd \& Silber. 2000: Golubitsky \& Stewart, 2002]. Central place theory in

\footnotetext{
${ }^{1} \mathrm{D}_{6}$ is the dihedral group expressing local hexagonal symmetry and $\mathbb{Z}_{n}$ is a cyclic group.
} 
economic geography was developed based on the distribution of cities and towns in Southern Germany [Christaller, 1933]. Eaton and Lipsey [1975] showed the existence of a hexagonal distribution of mobile production factors (e.g. firms and workers) based on a microeconomic foundation. Extensive studies of spatial agglomeration have been conducted on a square lattice Clarke \& Wilson, 1983; Weidlich \& Haag, 1987; Brakman et al., 1999]. The geometry of cities was explained by fractal [Batty \& Longley, 1994]. The distributions of satellite cities, towns, and villages around Berlin and London were shown to follow a universal law [Makse et al., 1995]. Self-organization in spatial systems was studied Banaszak et al., 2015] and a group-theoretic spectrum analysis of self-organization patterns of city distribution in Southern Germany and Eastern USA was conducted [Ikeda et al., 2018d].

This paper is organized as follows: A spatial economic model with the replicator dynamics is presented in Sec. 2 A hexagonal lattice and its bifurcating patterns are advanced in Sec. 3. A theory of invariant solutions of the replicator dynamics is introduced and is employed to obtain invariant solutions for a hexagonal lattice in Sec. 4. Numerical bifurcation analysis of a spatial economic model on the hexagonal lattice is carried out in Sec. 5 .

\section{Spatial Economic Model with the Replicator Dynamics}

A spatial economic model with the replicator dynamics is presented and its stationary points are classified. Whereas the theoretical framework of this paper is targeted for general spatial economic models, the FO model [Forslid \& Ottaviano, 2003] is employed as a concrete example and is to be used in the investigation of stability of agglomeration patterns (Sec. 5 ).

\subsection{Spatial economic model}

A spatial economic model of $K$ places is presented. We define the utility function vector $\mathbf{v} \in \mathbb{R}_{+}^{K}$ as a continuous function in the population distribution vector $\boldsymbol{\lambda}\left(\lambda_{i} \geq 0 ; i=1, \ldots, K\right)$ and the trade freeness parameter $\phi$. Workers or firms, footloose entrepreneurs, choose where to locate from $K$ places (actions), and the utility or profit (payoff) from locating in region $i$ is determined by the spatial distribution of workers or firms in the economy through the function $v_{i}$. An equilibrium is defined as the spatial distribution of workers $\boldsymbol{\lambda}$ that satisfies the following conditions:

$$
\begin{cases}v^{*}-v_{i}(\boldsymbol{\lambda}, \phi)=0 & \text { if } \lambda_{i}>0 \\ v^{*}-v_{i}(\boldsymbol{\lambda}, \phi) \geq 0 & \text { if } \lambda_{i}=0\end{cases}
$$

and $\sum_{i=1}^{K} \lambda_{i}=1$, where $v^{*}$ denotes the equilibrium utility level.

The utility is defined by the FO model among many alternatives. This model is briefly introduced here. There are two factors of production and two sectors. The two factors are skilled and unskilled labor and the workers supply one unit of each type of labor inelastically. The total endowment of skilled and unskilled workers is $H(=1)$ and $L(=1)$, respectively. The skilled worker is mobile across places and $\lambda_{i}$ denotes their number located in place $i$. The unskilled worker is immobile and equally distributed across all places (i.e. the number of unskilled workers in each place is $L / K)$. The two sectors consist of agriculture (abbreviated by A) and manufacturing (abbreviated by $M$ ). The A-sector output is homogeneous and each unit is produced using a unit of unskilled labor under perfect competition. The M-sector output is a horizontally differentiated product that is produced using both skilled and unskilled labor under increasing returns to scale and Dixit-Stiglitz monopolistic competition. There are two major parameters for this model: $\sigma$ expresses the constant elasticity of substitution between any two manufactured goods and $\mu$ denotes the constant expenditure shared on industrial varieties.

The goods of both sectors are transported. The transportation of A-sector goods is frictionless (cost free), while the transportation of M-sector goods demands iceberg costs. That is, for each unit of M-sector goods transported from place $i$ to $j(\neq i)$, only a fraction $1 / \tau_{i j}<1$ arrives. We assume that $\tau_{i i}=1$ for all $i \in\{1, \ldots, K\}$ and that $\tau_{i j}=\tau_{i j}(\tau)$ is a function in a transportation cost parameter $\tau>0$ as

$$
\tau_{i j}=\exp (\tau m(i, j))
$$

where $m(i, j)$ expresses the road distance between places $i$ and $j$ along the lattice.

The trade freeness is expressed by

$$
\phi=\exp [-(\sigma-1) d \tau], \quad \phi \in(0,1),
$$

where $d$ is the nominal length. 


\subsection{Replicator dynamics}

It is customary in economics to replace the problem to obtain a set of stable spatial equilibria by another problem to find a set of stable stationary points of the replicator dynamics:

$$
\frac{d \boldsymbol{\lambda}}{d t}=\mathbf{F}(\boldsymbol{\lambda}, \phi),
$$

where $\mathbf{F}(\boldsymbol{\lambda}, \phi)=\left(F_{i}(\boldsymbol{\lambda}, \phi) \mid i=1, \ldots, K\right)$, and

$$
F_{i}(\boldsymbol{\lambda}, \phi)=\left(v_{i}(\boldsymbol{\lambda}, \phi)-\bar{v}(\boldsymbol{\lambda}, \phi)\right) \lambda_{i} .
$$

Here, $\bar{v}=\sum_{i=1}^{K} \lambda_{i} v_{i}$ is the average utility. Stationary points (rest points) $\boldsymbol{\lambda}^{*}(\phi)$ of the replicator dynamics (4) are defined as those points which satisfy the static governing equation

$$
\mathbf{F}\left(\boldsymbol{\lambda}^{*}, \phi\right)=\mathbf{0} .
$$

Using the eigenvalues of the Jacobian matrix $J\left(\boldsymbol{\lambda}^{*}, \phi\right)=\partial \mathbf{F} / \partial \boldsymbol{\lambda}\left(\boldsymbol{\lambda}^{*}, \phi\right)$, we classify stability as

$$
\left\{\begin{array}{l}
\text { linearly stable: } \quad \begin{array}{rl}
\text { every eigenvalue has } \\
\text { a negative real part, }
\end{array} \\
\text { linearly unstable: } \begin{array}{c}
\text { at least one eigenvalue has } \\
\text { a positive real part. }
\end{array}
\end{array}\right.
$$

A stationary point is asymptotically stable or unstable according to whether it is linearly stable or unstable.

\subsection{Classifications of stationary points}

We advance the classifications of stationary points $(\boldsymbol{\lambda}, \phi)$ of the replicator dynamics. First, these points are classified into an interior solution, for which all cities have positive population, and a corner solution, for which some cities have zero population. A corner solution can be expressed, without loss of generality, by appropriately rearranging the order of independent variables $\boldsymbol{\lambda}$ as

$$
\hat{\lambda}=\left[\begin{array}{l}
\lambda_{+} \\
\lambda_{0}
\end{array}\right]
$$

with $\boldsymbol{\lambda}_{+}=\left\{\lambda_{i}>0 \mid i=1, \ldots, m\right\}$ and $\boldsymbol{\lambda}_{0}=\mathbf{0}$.

Next, stationary points are classified into an invariant solution and a noninvariant solution $(\boldsymbol{\lambda}, \phi)$. Stationary points that satisfy the static governing equation $\mathbf{F}(\boldsymbol{\lambda}, \phi)=\mathbf{0}$ form solution curves $(\boldsymbol{\lambda}(\phi), \phi)$ parameterized by $\phi$. In general, the spatial pattern $\boldsymbol{\lambda}(\phi)$ varies with $\phi$ along a solution curve. By contrast, there can be a special solution curve $(\boldsymbol{\lambda}(\phi), \phi)=(\overline{\boldsymbol{\lambda}}, \phi)$ that has a constant spatial pattern $\boldsymbol{\lambda}(\phi)=\overline{\boldsymbol{\lambda}}$ along the curve, as observed in the two-place economy Fujita et al., 1999]. Such pattern $\bar{\lambda}$ is called herein an invariant pattern. The curve of an invariant pattern exists for any $\phi \in(0,1)$. In contrast, a pattern $\boldsymbol{\lambda}(\phi)$ that varies with $\phi$ is called a noninvariant pattern and might or might not be a solution for a given $\phi$.

A special form of corner solution:

$$
\hat{\boldsymbol{\lambda}}=\left[\begin{array}{c}
\boldsymbol{\lambda}_{+} \\
\boldsymbol{\lambda}_{0}
\end{array}\right]=\left[\begin{array}{c}
\frac{1}{m} \mathbf{1} \\
\mathbf{0}
\end{array}\right]
$$

is advanced as the candidate of an invariant pattern. This is a core-periphery pattern with a twolevel hierarchy: population is agglomerated to $m$ core places with identical population, while other peripheral places have no populations.

There is a special kind of critical point, called sustain point, at which the population of some place(s) vanish(es). The associated equilibrium curve has a kink at this point.

\section{A Hexagonal Lattice and Its Bifurcating Patterns}

A hexagonal lattice is employed as a spatial platform for the spatial economic model (Sec. 2). After the introduction of the symmetry group of this lattice, examples of bifurcating patterns are presented.

\subsection{Hexagonal lattice and the group expressing its symmetry}

We consider a rhombic, $n \times n$ hexagonal lattice with periodic boundary conditions: an example for $n=2$ is shown by the dashed lines in Fig. 2. A system of

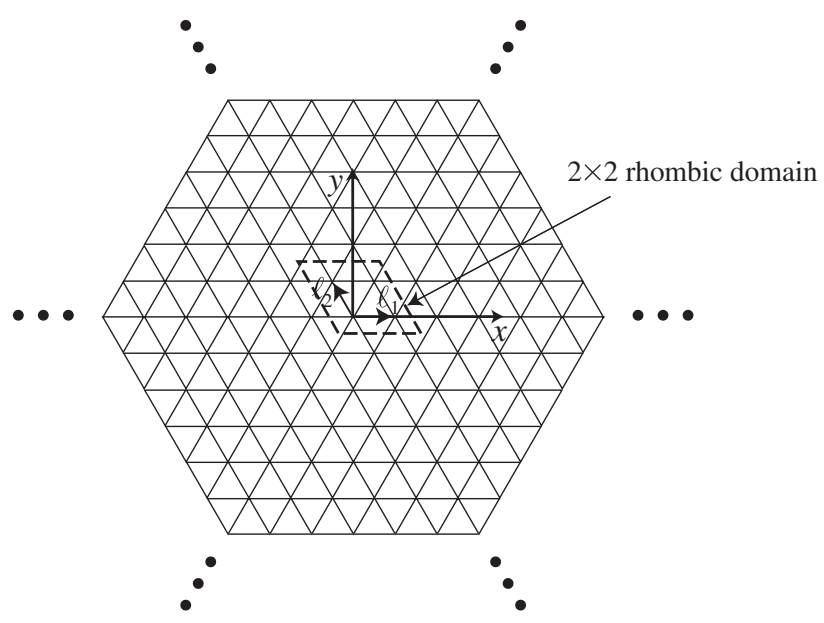

Fig. 2. Hexagonal lattice. 
$n \times n$ places is allocated at hexagonal lattice points

$$
\mathbf{p}=n_{1} \ell_{1}+n_{2} \ell_{2} \quad\left(n_{1}, n_{2}=0,1, \ldots, n-1\right)
$$

in a finite two-dimensional domain, where $\ell_{1}=$ $(d, 0)^{\top}$ and $\ell_{2}=(-d / 2, d \sqrt{3} / 2)^{\top}$ are oblique basis vectors ( $d$ is the length of these vectors). Neighboring places, by the periodic boundary conditions, are connected by straight lines of an equal length $d$ to form a regular-triangular mesh. These straight lines are interpreted as roads for the transportation of goods in the spatial economic model (Sec. 2).

Symmetry of the $n \times n$ hexagonal lattice is described by the group

$$
G=\left\langle r, s, p_{1}, p_{2}\right\rangle=\mathrm{D}_{6} \dot{+}\left(\mathbb{Z}_{n} \times \mathbb{Z}_{n}\right),
$$

where $\langle\cdots\rangle$ denotes a group generated by the elements therein, $\dot{+}$ expresses the semidirect product, and

- $r$ : counterclockwise rotation about the origin at an angle of $\pi / 3$.

- $s$ : reflection $y \mapsto-y$.
- $p_{1}$ : periodic translation along the $\boldsymbol{\ell}_{1}$-axis (i.e. the $x$-axis).

- $p_{2}$ : periodic translation along the $\boldsymbol{\ell}_{2}$-axis.

The group $G$ contains the dihedral group $\langle r, s\rangle \simeq \mathrm{D}_{6}$ and cyclic groups $\left\langle p_{1}\right\rangle \simeq \mathbb{Z}_{n}$ and $\left\langle p_{2}\right\rangle \simeq \mathbb{Z}_{n}$ as its subgroups, where $\simeq$ denotes that the associated two groups are isomorphic.

\subsection{Bifurcating patterns for spatial economic models}

We consider a scalar field on the hexagonal lattice for the spatial economic model in Sec. 2 The bifurcation of this model was studied and the eigenvectors of the Jacobian matrix associated with bifurcating solutions were obtained Ikeda \& Murota, 2014; Ikeda et al., 2018a. Examples of these eigenvectors for the $6 \times 6$ hexagonal lattice are depicted in Fig. 3. The eigenvectors express hexagonal and stripe patterns.
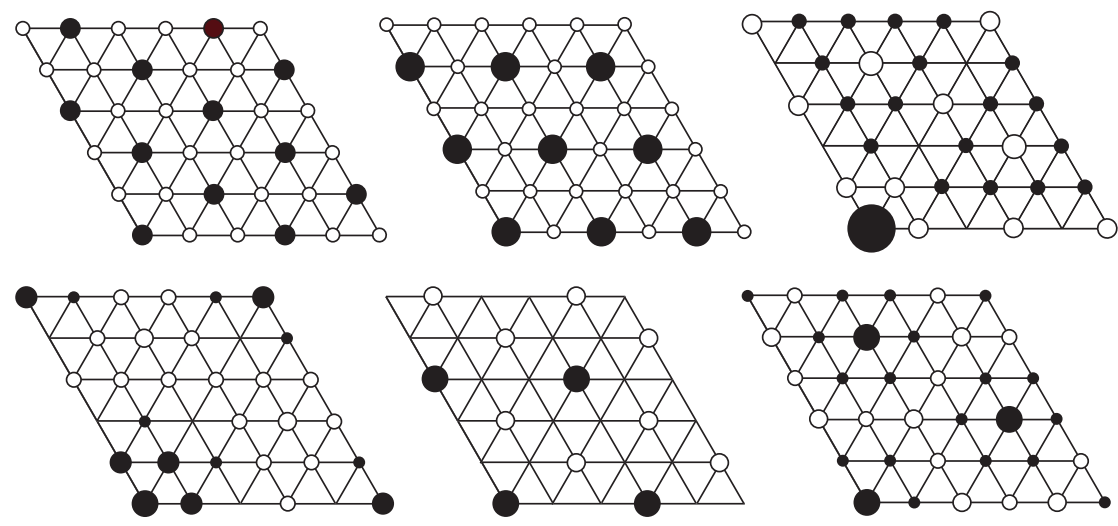

Hexagons
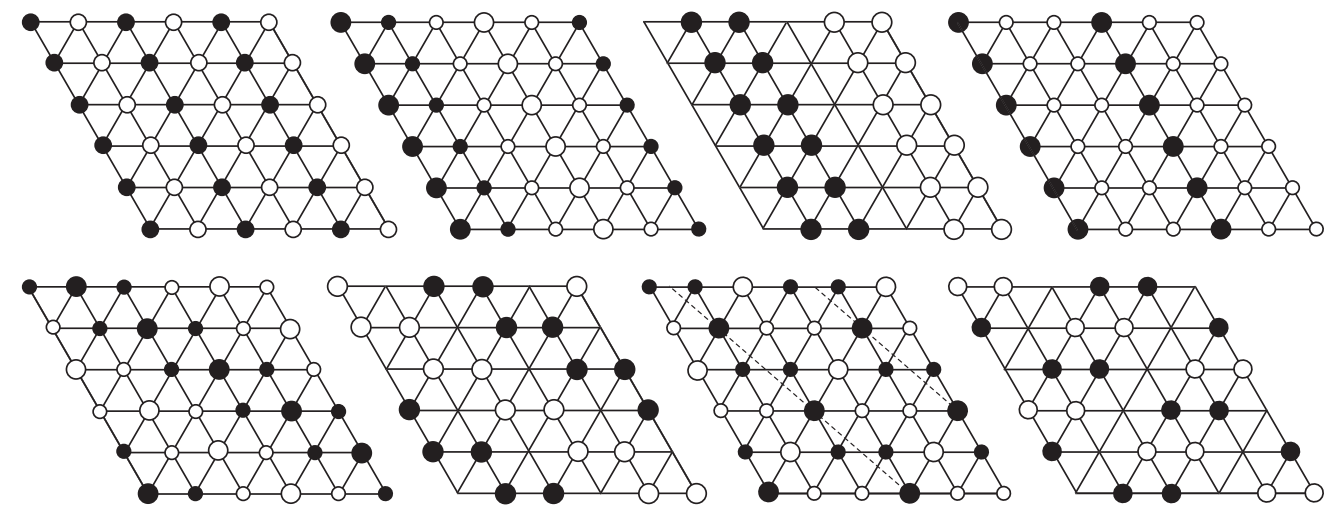

Stripes

Fig. 3. Hexagonal and stripe patterns expressed by the eigenvectors for bifurcating patterns on the $6 \times 6$ hexagonal lattice. The black circles indicate positive components; the white circles indicate negative components. 


\section{Invariant Patterns on the Hexagonal Lattice}

A theory of invariant patterns of the replicator dynamics is introduced and is employed to obtain invariant patterns on the hexagonal lattice.

\subsection{Theory}

As candidates of invariant patterns, we consider the spatial pattern of the corner solution $\left(\boldsymbol{\lambda}_{+}, \boldsymbol{\lambda}_{0}, \phi\right)=$ $\left(\frac{1}{m} \mathbf{1}, \mathbf{0}, \phi\right)\left(1 \leq m<n^{2}\right)$ in (8) $)$, expressing equal complete agglomeration to $m$ places. This pattern can be an invariant pattern under some symmetry conditions, as explained in the following proposition (see Appendix A for the proof).

Proposition 1. A spatial pattern $\left(\boldsymbol{\lambda}_{+}, \boldsymbol{\lambda}_{0}\right)=\left(\frac{1}{m} \mathbf{1}\right.$, $\mathbf{0})$ is an invariant pattern if this pattern satisfies

(1) $\left(\boldsymbol{\lambda}_{+}, \boldsymbol{\lambda}_{0}\right)=\left(\frac{1}{m} \mathbf{1}, \mathbf{0}\right)$ is invariant to a group $G^{\prime}$.

(2) The set of points for $\boldsymbol{\lambda}_{+}$belongs to an orbit.

In particular, the patterns for $m=1, m=$ 2 , and $m=n^{2}$ are called (atomic) monocenter, twin, and uniform, respectively and play pivotal roles throughout this paper 2 We have the following propositions for these patterns (see Appendix A for the proof).

Proposition 2. A monocenter at any place is an invariant solution for any economy.

Proposition 3. The uniform state and twins on a hexagonal lattice are invariant patterns.

\subsection{Procedure to obtain invariant patterns}

We can search for invariant patterns by finding a set of $m$ nodal points and a subgroup $G^{\prime}$ of the group $G=\left\langle r, s, p_{1}, p_{2}\right\rangle$ in (9) that satisfy Proposition [1. We propose the following procedure to obtain all invariant patterns on the hexagonal lattice with given $n(\geq 3)$ and $m\left(1 \leq m \leq n^{2}\right)$.

- Choose a set of $m$ nodal points among a total of $n^{2}$ nodal points.

- Find elements of $G$ that keep the set of points invariant.

- If these elements form a group and permute any two of the $m$ nodal points, this group is chosen as $G^{\prime}$ in Lemma 1 to ensure that the set of points gives an invariant pattern.

In the procedure, we already know that the monocenters $(m=1)$, the twins $(m=2)$, and the uniform state $\left(m=n^{2}\right)$ are invariant patterns by Propositions 2 and 3 , therefore, we focus on $3 \leq m<n^{2}$. Moreover, the number $m$ is not arbitrary but is dependent on the lattice size $n$ as explained in Proposition 4 (see Appendix A for the proof). Table 1 lists possible values of $m$, e.g. for $n=3,4,5,6$.

Table 1. Possible values of the number $m$ for several lattice sizes $n$ ( $\mathbb{Z}$ is a set of integer numbers).

\begin{tabular}{|c|c|c|c|}
\hline$n$ & $12 n^{2}$ & $m$ & $\overline{12 n^{2} / m \in \mathbb{Z}}$ \\
\hline 3 & 108 & $\begin{array}{l}1 \\
2 \\
3 \\
4 \\
6 \\
9\end{array}$ & $\begin{array}{r}108 \\
54 \\
36 \\
27 \\
18 \\
12\end{array}$ \\
\hline 4 & 192 & $\begin{array}{r}1 \\
2 \\
3 \\
4 \\
6 \\
8 \\
12 \\
16\end{array}$ & $\begin{array}{r}192 \\
96 \\
64 \\
48 \\
32 \\
24 \\
16 \\
12\end{array}$ \\
\hline 5 & 300 & $\begin{array}{r}1 \\
2 \\
3 \\
4 \\
5 \\
6 \\
10 \\
25\end{array}$ & $\begin{array}{r}300 \\
150 \\
100 \\
75 \\
60 \\
50 \\
30 \\
12\end{array}$ \\
\hline 6 & 432 & $\begin{array}{r}1 \\
2 \\
3 \\
4 \\
6 \\
8 \\
9 \\
12 \\
18 \\
24 \\
27 \\
36\end{array}$ & $\begin{array}{r}432 \\
216 \\
144 \\
108 \\
72 \\
54 \\
48 \\
36 \\
24 \\
18 \\
16 \\
12\end{array}$ \\
\hline
\end{tabular}

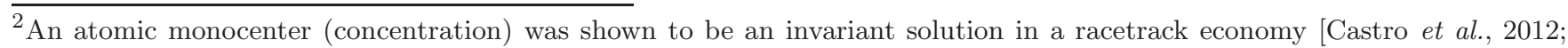
Ikeda et al., 2012a. 


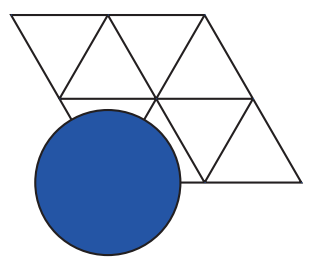

$$
G^{\prime}=\langle s, r\rangle
$$$$
m=1
$$

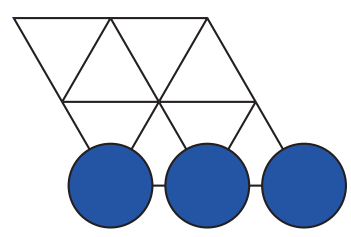

$$
G^{\prime}=\left\langle s, p_{1}, r^{3}\right\rangle
$$

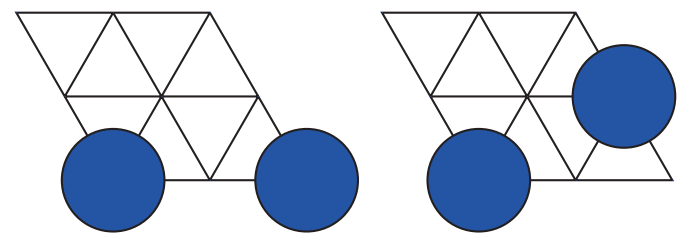

$$
G^{\prime}=\left\langle s, p_{1} r^{3}\right\rangle \quad G^{\prime}=\left\langle r s, p_{1}^{2} p_{2} r^{3}\right\rangle
$$

$m=2$
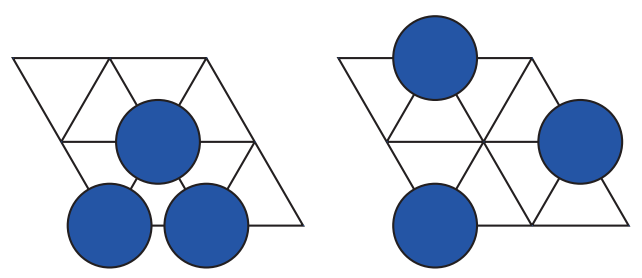

$$
\begin{gathered}
G^{\prime}=\left\langle r s, p_{1} s r^{3}\right\rangle \\
m=3
\end{gathered}
$$$$
G^{\prime}=\left\langle s, r, p_{1}^{2} p_{2}\right\rangle
$$

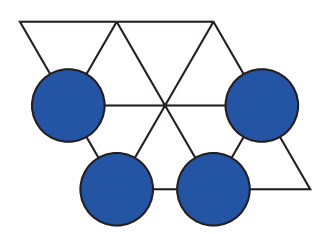

$$
G^{\prime}=\left\langle s p_{1} p_{2}^{2}, r^{3} p_{2}^{2}\right\rangle
$$$$
m=4
$$
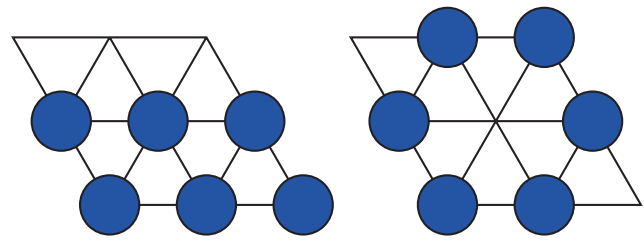

$G^{\prime}=\left\langle s r^{3}, p_{2} r^{3}, p_{1}\right\rangle$

$G^{\prime} \simeq\left\langle s, r, p_{1}^{2} p_{2}\right\rangle$

$m=6$

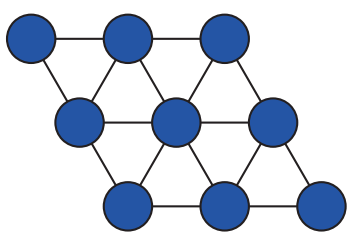

$G^{\prime}=\left\langle s, r, p_{1}, p_{2}\right\rangle$

$m=9$

Fig. 4. Invariant patterns for $n=3$.

Proposition 4. The number $m\left(1 \leq m \leq n^{2}\right)$ divides $12 n^{2}$.

Figure 4 depicts all invariant patterns for the lattice size $n=3$ obtained in this manner, whereas those for $n=4,5,6$ are presented in Appendix B. Invariant patterns that exist for any $n$ are listed in Fig. 5. These patterns include a triangle, racetrack, and stripe, in addition to a monocenter, twin, and uniform, which are proved as invariant patterns above.

\subsection{Invariant patterns engendered by direct bifurcations}

We investigate the connection of invariant patterns to bifurcating patterns from the uniform state. Recall the plots of critical eigenvectors of bifurcating patterns in Fig. 3. We assume that population is agglomerated completely to place(s) with the largest positive or negative component(s) of these eigenvectors and population elsewhere vanishes; this assumption is fairly in line with the agglomeration behavior for a spatial economic model to be observed in Sec. 5. Under this assumption, we see that the invariant patterns shown in Fig. [6] can possibly be engendered by the direct bifurcation. However, it is noteworthy that not all invariant patterns can be engendered by the bifurcation. The existence of nonbifurcation oriented invariant patterns makes agglomeration behaviors very complicated (Sec. 5).

\section{Numerical Bifurcation and Stability Analysis for a Spatial Economic Model}

A numerical bifurcation and stability analysis is conducted on $3 \times 3$ and $6 \times 6$ hexagonal lattices for a spatial economic model Forslid \& Ottaviano, 2003. Parameter values are chosen as $(\sigma, \mu)=(8.0$, $0.4)$ and the distance unit as $d=1 / n(n=3,6)$. 


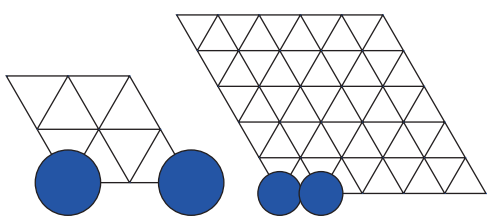

$G^{\prime}=\left\langle s, p_{1} r^{3}\right\rangle$

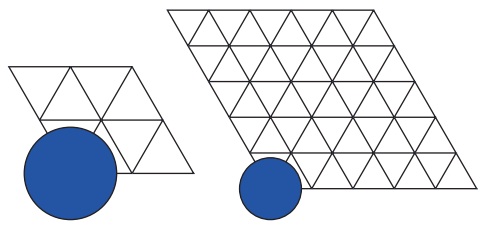

$G^{\prime}=\langle s, r\rangle$

Monocenters

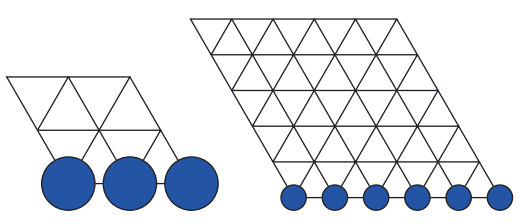

$G^{\prime}=\left\langle s, p_{1}, r^{3}\right\rangle$

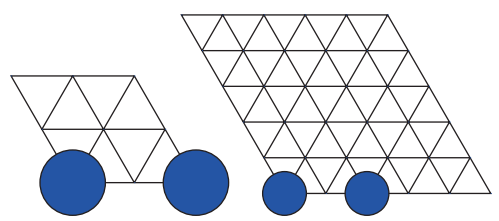

$G^{\prime}=\left\langle s, p_{1}^{2} r^{3}\right\rangle$

Twins

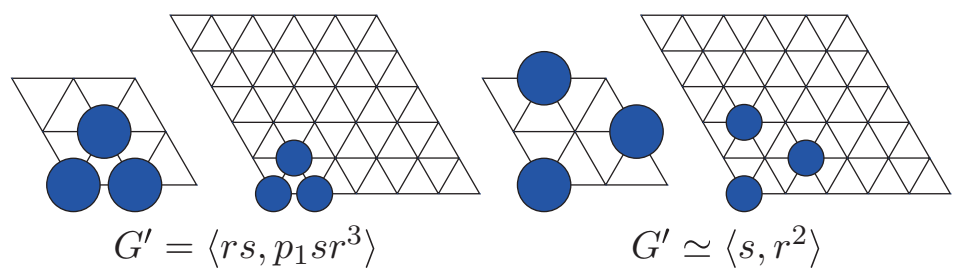

Triangles

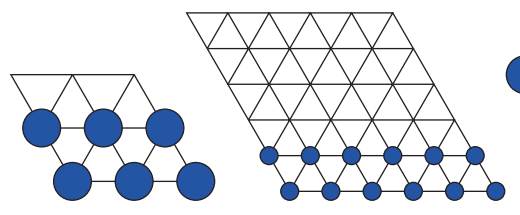

$G^{\prime}=\left\langle s r^{3}, p_{2} r^{3}, p_{1}\right\rangle$

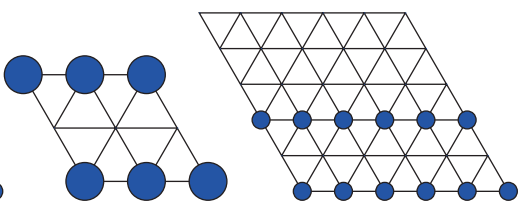

$G^{\prime}=\left\langle s r^{3}, p_{2}^{2} r^{3}, p_{1}\right\rangle$

Stripes

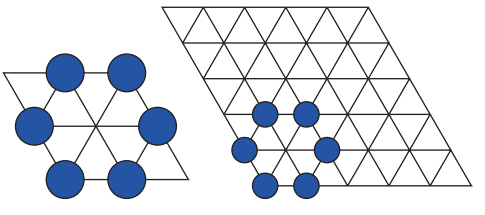

$G^{\prime} \simeq\langle s, r\rangle$

Racetracks

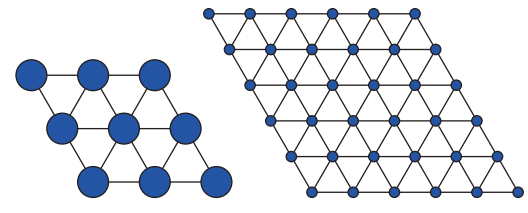

$G^{\prime}=\left\langle s, r, p_{1}, p_{2}\right\rangle$

Uniform

Fig. 5. Invariant patterns that exist for any $n(n \geq 3)$.

As a procedure to search for stable equilibrium curves numerically, we propose the following innovative procedure that exploits the existence of invariant solutions:

- Obtain all invariant patterns and investigate their stability by the eigenanalysis of the Jacobian matrix for $\phi \in(0,1)$.

- Obtain bifurcating equilibrium curves connecting stable invariant solutions and investigate their stability.
This procedure is convenient in that we can start the bifurcation analysis from any stable invariant solutions, whereas it is conventional to start only from the uniform state.

\section{1. $3 \times 3$ hexagonal lattice}

The stability of the ten invariant patterns for the lattice size $n=3$ in Fig. 4 was investigated. Among these ten patterns, the six patterns shown in Fig. [7(a) were stable 3 in some ranges of $\phi$ shown

\footnotetext{
${ }^{3}$ Stripes and Triangles have exactly the same range of $\phi$ as these two invariant patterns have the same economical environment: an agglomerated place is connected to two other agglomerated places at a distance of $d$, four nonpopulated places at a distance of $d$, and two other nonpopulated places at a distance of $2 d$.
} 


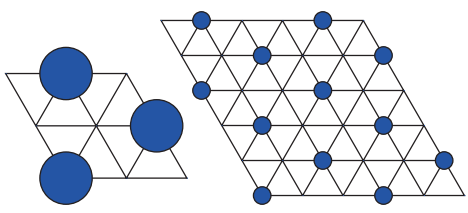

$k=3$

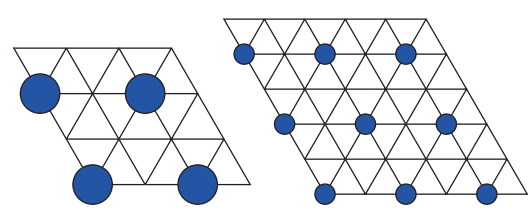

$k=4$

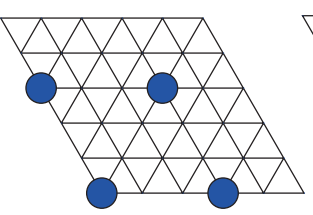

$k=9$

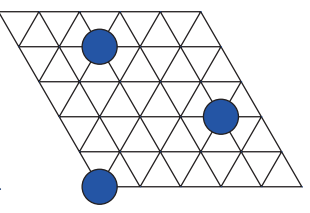

$k=12$

Hexagons
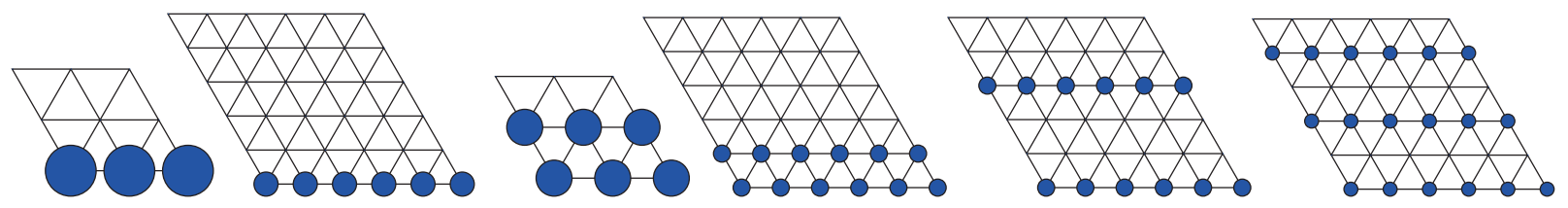

Stripes

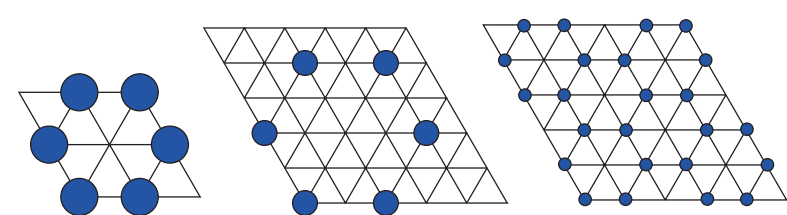

Racetracks

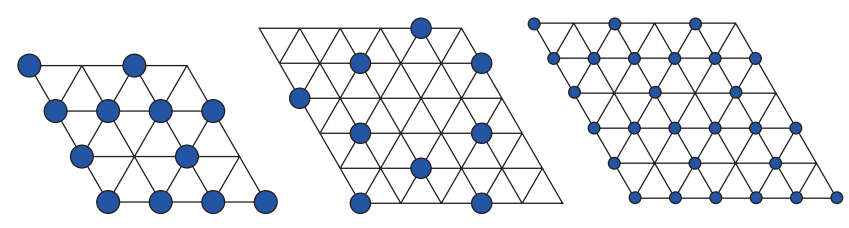

Hexagrams

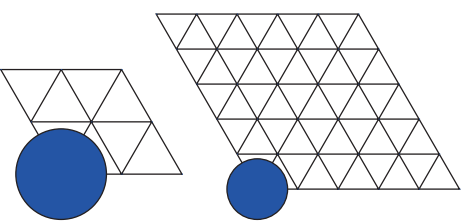

Monocenters

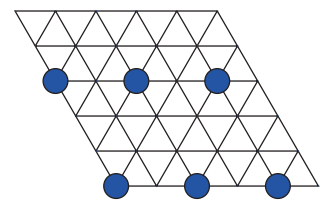

Parallelogram

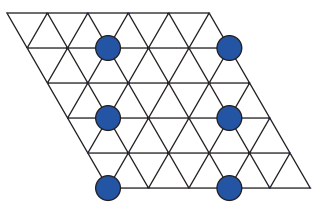

Rectangle

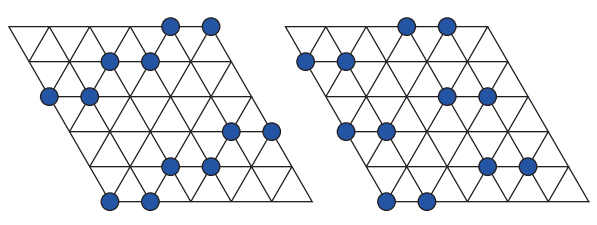

Wavy stripes

Fig. 6. Invariant solutions that can be engendered by the direct bifurcation from the uniform state.

by the solid lines: - There are bifurcation points 4 at both end points of the ranges, except for the end point $a$ with $\phi=0$ and the end point $n$ with $\phi=1$. Thus stable invariant patterns become unstable by bifurcations at end points as $\phi$ changes. The remaining four patterns shown in Fig. 7(b) were unstable for any $\phi \in(0,1)$.

Table 2(a) lists the number of stable and unstable invariant patterns classified with reference to the number $m$ of agglomerated places. An invariant pattern with a larger $m$ tends to become unstable (except for the uniform pattern with $m=9$ ). Also, from Fig. 7(a) we can see a tendency that an invariant pattern with a smaller $m$ tends to become stable as the trade freeness $\phi$ increases.

A bifurcation analysis was conducted to find the equilibrium curves for noninvariant patterns that connect the stable invariant patterns. In Fig. 7(c), the nonhorizontal curves display the equilibrium curves for noninvariant patterns and horizontal lines denote those for invariant ones; here stable lines and curves are shown by - and unstable ones by - - - These nonhorizontal curves were unstable, except near the point $d^{\prime}$. Thus noninvariant patterns are inferior in stability.

We can see a mesh-like structure made up of horizontal lines and nonhorizontal curves, just like threads of warp and weft. For example, the horizontal curves $a b$ and $c d$ for the uniform state and the flat line $e f$ for the hexagon $(k=3)$ are connected by nonhorizontal curves be and $c f$ of noninvariant patterns. As we have seen, a knowledge of invariant solutions is useful in obtaining that mesh-like structure.

\footnotetext{
${ }^{4}$ The multiplicity $M$ of these points is either $1,2,3$, or 6 , which is shown by the symbols $\times, \circ, \triangle$, or + , respectively.
} 
K. Ikeda et al.

(a)

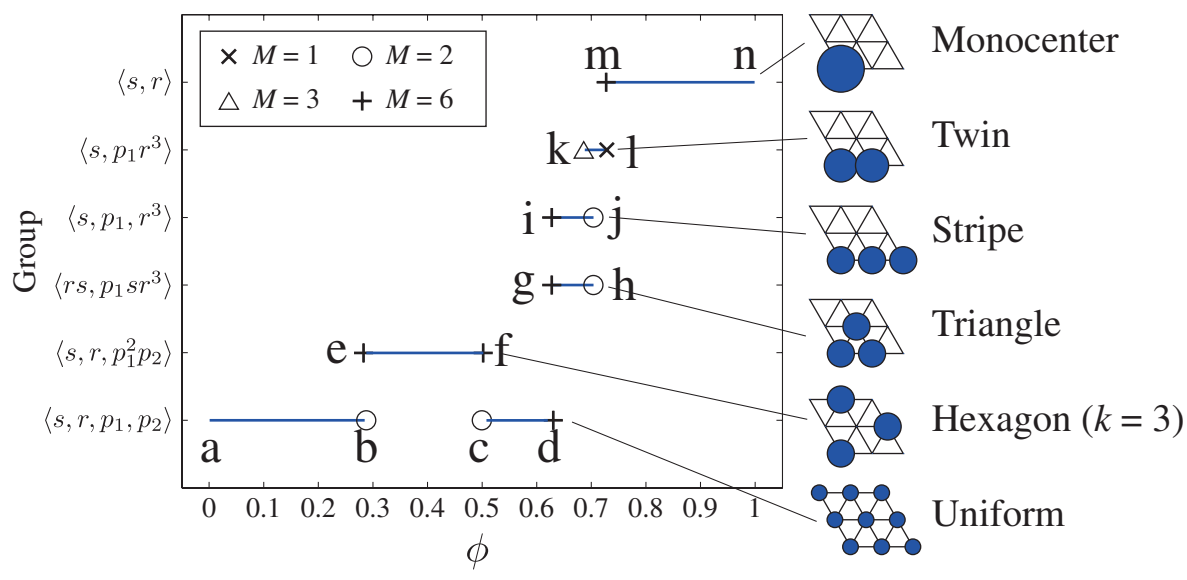

(b)

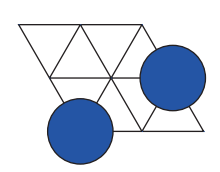

Twin (oblique)

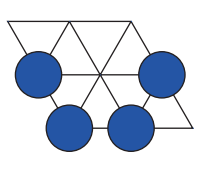

Wavy stripe

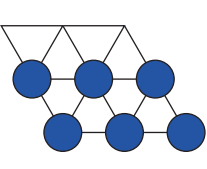

Stripe (double)

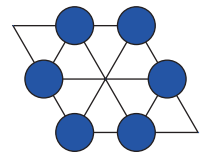

Racetrack

(c)

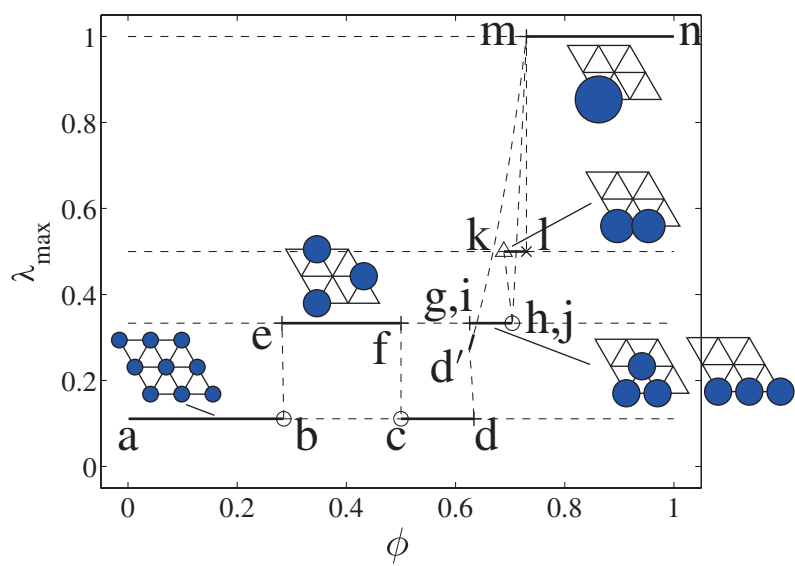

Fig. 7. Stable patterns for a $3 \times 3$ hexagonal lattice and their equilibrium paths. (a) The ranges of $\phi$ for stable invariant patterns, (b) unstable invariant patterns and (c) equilibrium curves of invariant and noninvariant patterns. $\lambda_{\max }=$ $\max \left(\lambda_{1}, \ldots, \lambda_{K}\right) ;-$ : stable curve; - - -: unstable curve.

Table 2. The number of stable and unstable invariant patterns classified with reference to the number $m$ of agglomerated places.

\begin{tabular}{r|rrrrrr}
\hline \multicolumn{7}{|c}{ (a) $n=3$} \\
\hline$m$ & 1 & 2 & 3 & 4 & 6 & 9 \\
\hline Stable & 1 & 1 & 3 & 0 & 0 & 1 \\
Unstable & 0 & 1 & 0 & 1 & 2 & 0 \\
\hline Total & 1 & 2 & 3 & 1 & 2 & 1 \\
\hline
\end{tabular}

(b) $n=6$

\begin{tabular}{r|rrrrrrrrrrrrr}
\hline$m$ & 1 & 2 & 3 & 4 & 6 & 8 & 9 & 12 & 16 & 18 & 24 & 27 & 36 \\
\hline Stable & 1 & 3 & 5 & 3 & 3 & 1 & 1 & 1 & 0 & 0 & 0 & 0 & 1 \\
Unstable & 0 & 3 & 2 & 8 & 10 & 5 & 2 & 9 & 1 & 3 & 2 & 1 & 0 \\
\hline Total & 1 & 6 & 7 & 11 & 13 & 6 & 3 & 10 & 1 & 3 & 2 & 1 & 1 \\
\hline
\end{tabular}




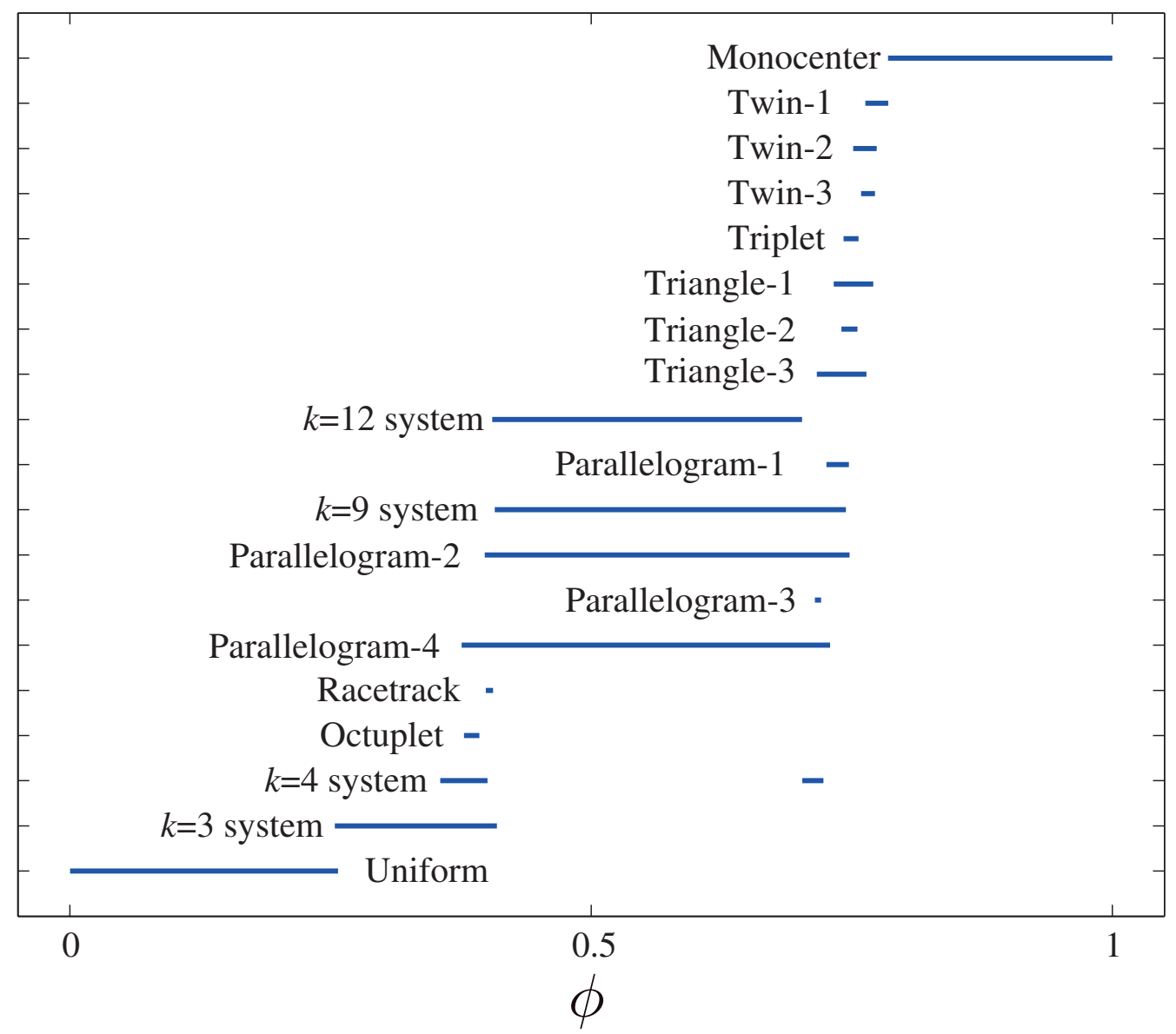

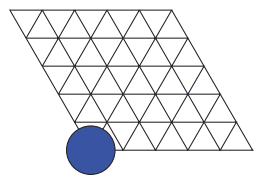

Monocenter

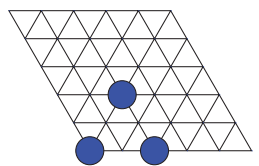

Triangle-1

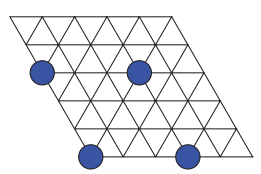

$k=9$ system

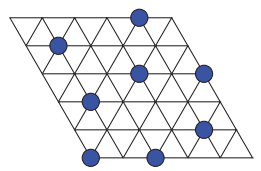

Octuplet

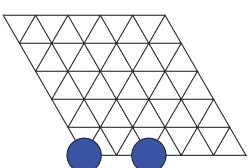

Twin-1

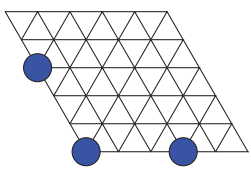

Triangle-2

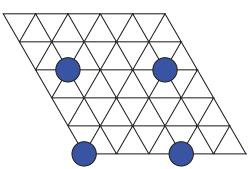

Parallelogram-2

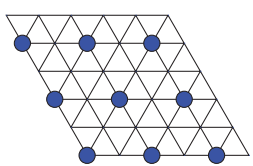

$k=4$ system

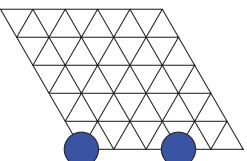

Twin-2

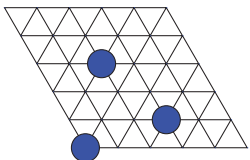

Triangle-3

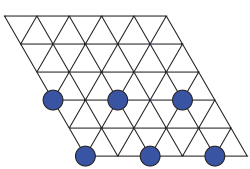

Parallelogram-3

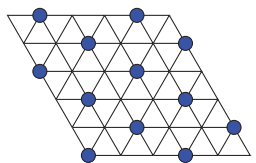

$k=3$ system

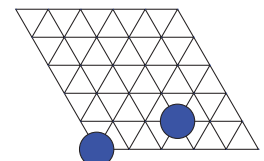

Twin-3

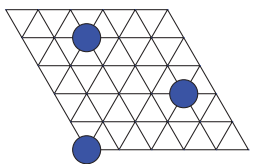

$k=12$ system

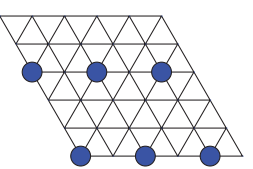

Parallelogram-4

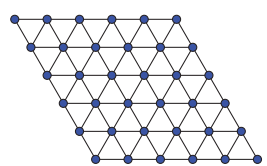

Uniform

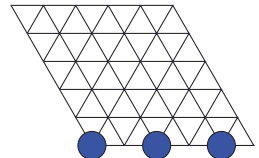

Triplet

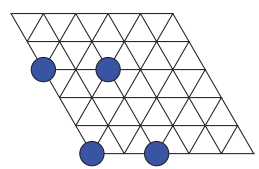

Parallelogram-1

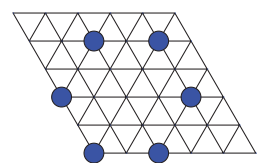

Racetrack

Fig. 8. The ranges of $\phi$ for stable invariant patterns for a $6 \times 6$ hexagonal lattice. 


\section{2. $6 \times 6$ hexagonal lattice}

The stability of all invariant patterns for the lattice size $n=6$ (Fig. 13 in Appendix B) was investigated. Invariant patterns that are stable for some $\phi$ are shown in Fig. 8. There are as many as 19 stable invariant patterns, whereas there are only six stable ones for $n=3$ [cf. Fig. 7 (a)]. It is expected that the number of stable invariant ones increases as the lattice size $n$ increases. As shown in Table 2(b), an invariant pattern with a larger $m$ tends to become unstable (except for the uniform pattern with $m=36$ ), similarly to the case of $n=3$.

The equilibrium paths shown in Fig. 9 were computed. As can be seen, there is a mesh-like structure of equilibrium paths made up of horizontal lines for invariant patterns and nonhorizontal
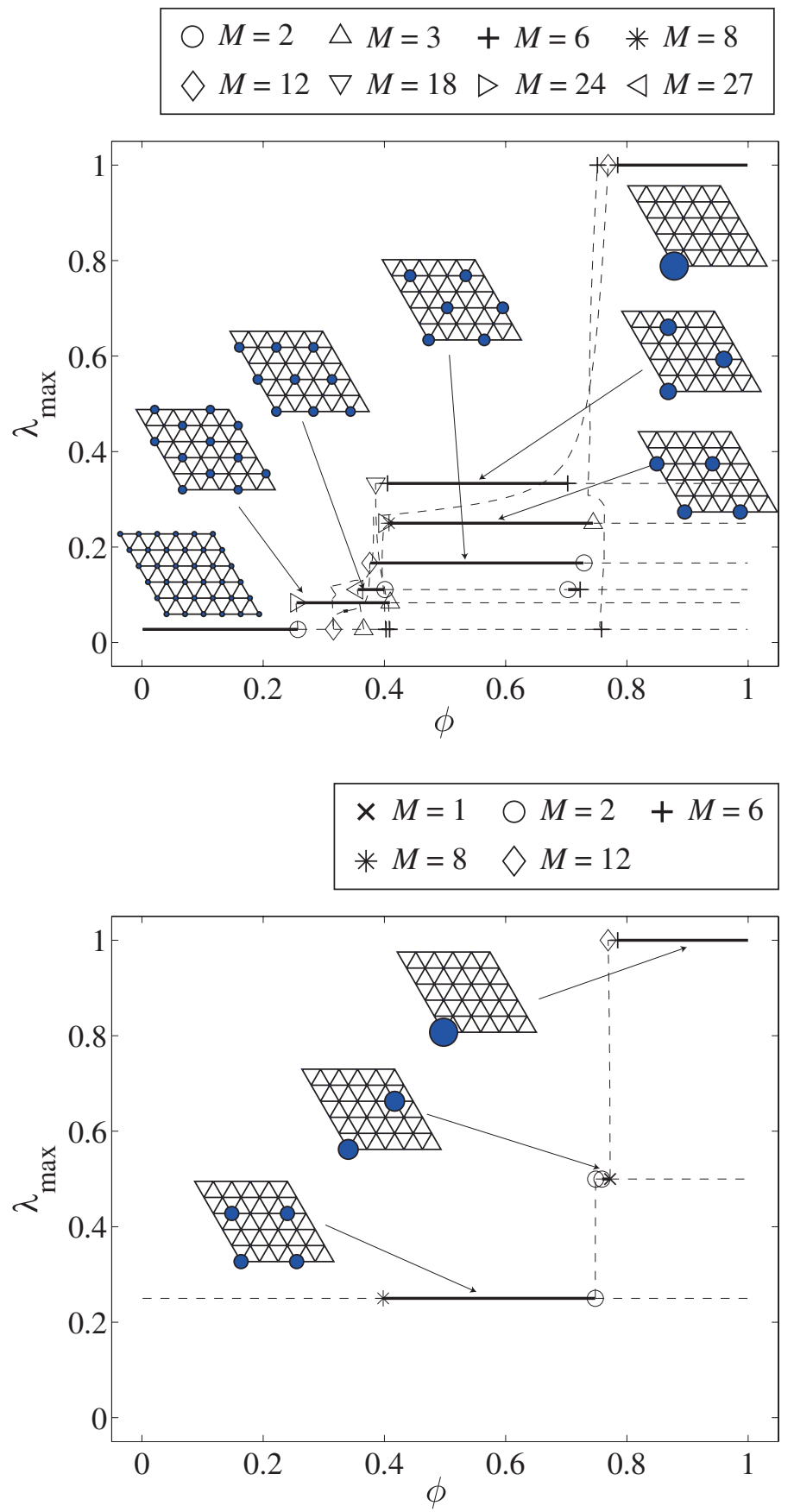

Fig. 9. Equilibrium curves of invariant and noninvariant patterns for a $6 \times 6$ hexagonal lattice. $\lambda_{\max }=\max \left(\lambda_{1}, \ldots, \lambda_{K}\right) ;-$ stable curve; - - -: unstable curve. 


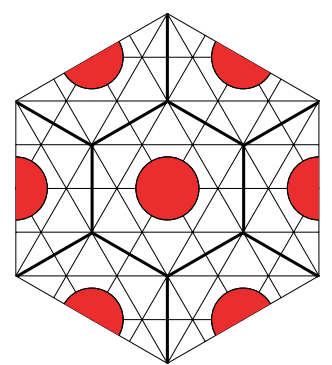

Hexagon $(k=12)$

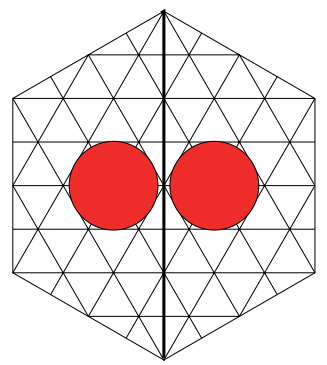

Twin-1

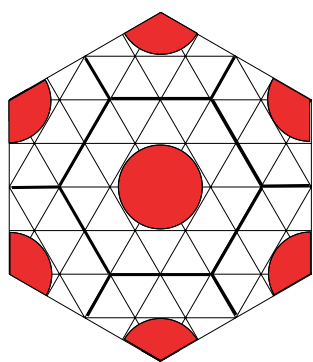

Hexagon $(k=9)$

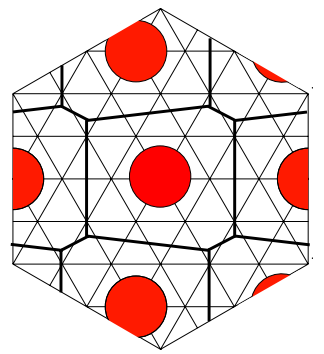

Parallelogram-2

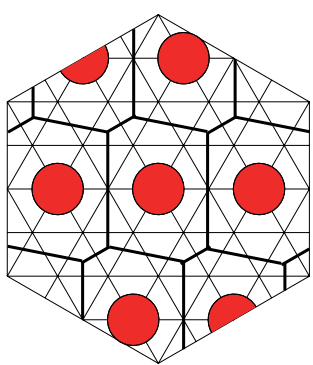

Parallelogram-4

(a)

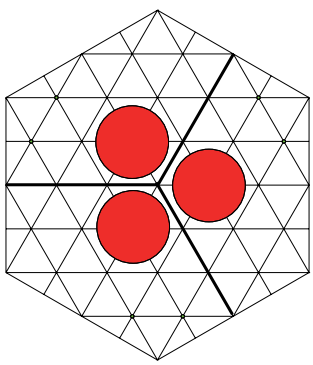

Triangle-1

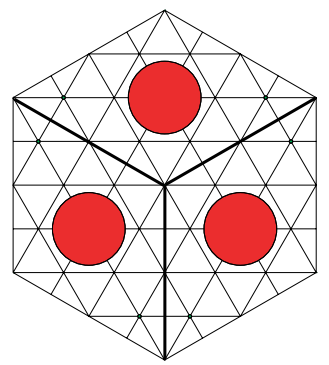

Triangle-2

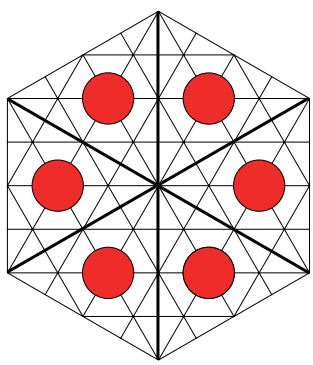

Racetrack

(b)

Fig. 10. Market areas for invariant patterns. (a) Patterns superior in stability and (b) patterns inferior in stability.

curves for noninvariant patterns. Again noninvariant patterns are inferior in stability as almost all the curves for noninvariant patterns are unstable.

In central place theory, each agglomerated place is considered to have a market area in its neighborhood. A place with no population belongs to the market area of the closest agglomerated place. Examples of market areas for invariant patterns are shown in Fig. 10, in which the border of a market area is shown by the bold solid line and hexagonal windows in this figure are cut from spatially repeated $6 \times 6$ rhombic domains with periodic boundaries. As can be seen, there are market areas of several shapes: hexagon, triangle, rhombus, rectangle, and so on. The patterns shown in Fig. 10(a) are superior in stability in the sense that they are stable for wide ranges of $\phi$ (Fig. 8). The market area for an agglomerated place has a regular-hexagonal or deformed-hexagonal shape. For the patterns inferior in stability shown in Fig. 10(b), a market area has a trapezoidal, crown-like, rhombic, or triangular shape. As we have seen, market areas closer to a circle are superior in stability in line with the discussion in central place theory Christaller, 1933; Lösch, 1954.

\section{Conclusions}

In spatial economic agglomerations on a hexagonal lattice, invariant patterns display characteristic geometrical patterns. This paper has conducted a group-theoretic analysis of these patterns. A methodology to find these patterns has been proposed and put to use to exhaustively obtain invariant patterns of the hexagonal lattice. Possible bifurcating patterns found herein were quite diverse, encompassing: monocenters, twins, triangles, rhombics, hexagons, racetracks, stripes, hexagrams, and so on.

The numerical bifurcation and stability analysis of invariant and noninvariant patterns were conducted. It was found that there are a number of stable invariant patterns, whereas there are few stable noninvariant patterns. Thus invariant patterns are much superior in stability. Such superiority ensures the usefulness and insightfulness of the bifurcation analysis procedure proposed herein that exploits the existence of invariant solutions.

We have found a mesh-like structure of equilibrium curves. There are horizontal lines for invariant patterns and nonhorizontal curves for noninvariant patterns, just like threads of warp and weft. 
It would be an important scientific mission to elucidate the mechanism of this complicated structure through the introduction of bifurcation mechanism in nonlinear mathematics. Such elucidation of the mechanism will contribute to the study of economic agglomerations.

\section{Acknowledgment}

Grant-in-Aid for JSPS 18K04380/18K18874 is greatly appreciated.

\section{References}

Akamatsu, T., Takayama, Y. \& Ikeda, K. [2012] "Spatial discounting, Fourier, and racetrack economy: A recipe for the analysis of spatial agglomeration models," $J$. Econ. Dyn. Contr. 36, 1729-1759.

Baldwin, R., Forslid, R., Martin, P., Ottaviano, G. \& Robert-Nicoud, F. [2003] Economic Geography and Public Policy (Princeton University Press, Princeton).

Banaszak, M., Dziecielski, M., Nijkamp, P. \& Ratajczak, W. [2015] "Self-organisation in spatial systems: From fractal chaos to regular patterns and vice versa," PLoS One 10, e0136248.

Batty, M. \& Longley, P. [1994] Fractal Cities (Academic Press, San Diego).

Bénard, H. [1900] "Les tourbillons cellulaires dans une nappe liquide," Rev. Gén. Sci. Pure Appl. 11, 12611271, 1309-1328.

Brakman, S., Garretsen, H., van Marrewijk, C. \& van den Berg, M. [1999] "The return of Zipf: Towards a further understanding of the rank-size distribution," J. Reg. Sci. 39, 183-213.

Buzano, E. \& Golubitsky, M. [1983] "Bifurcation on the hexagonal lattice and the planar Bénard problem," Philos. Trans. Roy. Soc. Lond. A 308, 617-667.

Castro, S. B. S. D., Correia-da-Silva, J. \& Mossay, P. [2012] "The core-periphery model with three regions and more," Pap. Reg. Sci. 91, 401-418.

Christaller, W. [1933] Die zentralen Orte in Süddeutschland (Gustav Fischer, Jena); English translation [1966] Central Places in Southern Germany (Prentice Hall, Englewood Cliffs).

Clarke, M. \& Wilson, A. G. [1983] "The dynamics of urban spatial structure: Progress and problems," $J$. Reg. Sci. 23, 1-18.

Dionne, B. \& Golubitsky, M. [1992] "Planforms in two and three dimensions," Z. Angew. Math. Phys. 43, $36-62$.

Dionne, B., Silber, M. \& Skeldon, A. C. [1997] "Stability results for steady, spatially, periodic planforms," Nonlinearity 10, 321-353.
Eaton, B. C. \& Lipsey, R. G. [1975] "The principle of minimum differentiation reconsidered: Some new developments in the theory of spatial competition," Rev. Econ. Stud. 42, 27-49.

Forslid, R. \& Ottaviano, G. I. P. [2003] "An analytically solvable core-periphery model," J. Econ. Geogr. 3, 229-340.

Fujita, M. \& Mori, T. [1997] "Structural stability and the evolution of urban systems," Reg. Sci. Urban Econ. 42, 399-442.

Fujita, M., Krugman, P. \& Venables, A. J. [1999] The Spatial Economy (MIT Press, Cambridge).

Golubitsky, M., Stewart, I. \& Schaeffer, D. G. [1988] Singularities and Groups in Bifurcation Theory, Vol. 2 (Springer, NY).

Golubitsky, M. \& Stewart, I. [2002] The Symmetry Perspective (Birkhäuser, Basel).

Ikeda, K., Akamatsu, T. \& Kono, T. [2012a] "Spatial period-doubling agglomeration of a core-periphery model with a system of cities," J. Econ. Dyn. Contr. 36, 754-778.

Ikeda, K., Murota, K. \& Akamatsu, T. [2012b] "Selforganization of Lösch's hexagons in economic agglomeration for core-periphery models," Int. J. Bifurcation and Chaos 22, 1230026-1-29.

Ikeda, K. \& Murota, K. [2014] Bifurcation Theory for Hexagonal Agglomeration in Economic Geography (Springer, Tokyo).

Ikeda, K., Aizawa, H., Kogure, Y. \& Takayama, Y. [2018a] "Stability of bifurcating patterns of spatial economy models on a hexagonal lattice," Int. J. Bifurcation and Chaos 28, 1850138-1-30.

Ikeda, K., Onda, M. \& Takayama, Y. [2018b] "Spatial period doubling, invariant pattern, and break point in economic agglomeration in two dimensions," J. Econ. Dyn. Contr. 92, 129-152.

Ikeda, K., Onda, M. \& Takayama, Y. [2018c] "Bifurcation theory of a racetrack economy in a spatial economy model," Netw. Spat. Econ., https://doi.org/10.1007/s11067-018-9423-0.

Ikeda, K., Takayama, Y., Onda, M. \& Murakami, D. [2018d] "Group-theoretic spectrum analysis of population distribution in Southern Germany and Eastern USA," Int. J. Bifurcation and Chaos 28, 1830045-127.

Judd, S. L. \& Silber, M. [2000] "Simple and superlattice turning patterns in reaction-diffusion systems: Bifurcation, bistability, and parameter collapse," Physica D 136, 45-65.

Kirchgässner, K. [1979] "Exotische Lösungen Bénardschen problems," Math. Meth. Appl. Sci. 1, 453-467.

Kochendörffer, R. [1970] Group Theory (McGraw-Hill, London).

Lösch, A. [1954] The Economics of Location (Yale University Press, London). 
Makse, H. A., Havlin, S. \& Stanley, H. E. [1995 "Modelling urban growth patterns," Nature 377, 608-612.

Mori, T. [1997] "A modeling of megalopolis formation: The maturing of city systems," J. Urban Econ. 42, 133-157.

Sandholm, W. H. [2010] Population Games and Evolutionary Dynamics (MIT Press, Cambridge).

Sattinger, D. H. [1978] "Group representation theory, bifurcation theory and pattern formation," J. Funct. Anal. 28, 58-101.

Tabuchi, T. \& Thisse, J. F. [2011] "A new economic geography model of central places," J. Urban Econ. 69, 240-252.

Weidlich, W. \& Haag, G. [1987] "A dynamic phase transition model for spatial agglomeration processes," $J$. Reg. Sci. 27, 529-569.

\section{Appendix A}

\section{Proofs of Propositions}

Proof of Proposition 1. Since the $m$ places of $\boldsymbol{\lambda}_{+}$ belong to an orbit, we have $v_{1}=\cdots=v_{m}$, as well as $\lambda_{1}=\cdots=\lambda_{m}=1 / m$. Then we have

$$
\bar{v}=\sum_{i=1}^{m} \lambda_{i} v_{i}=v_{i}
$$

and, in turn, $v_{i}-\bar{v}=0(i=1, \ldots, m)$, thereby satisfying $\mathbf{F}_{+}\left(\frac{1}{m} \mathbf{1}, \mathbf{0}, \tau\right)=\mathbf{0}$. For $K-m$ places with no population $\left(K=n^{2}\right)$, we have $\lambda_{j}=0$, thereby satisfying $\mathbf{F}_{0}\left(\frac{1}{m} \mathbf{1}, \mathbf{0}, \tau\right)=\mathbf{0}$. This shows that $\left(\boldsymbol{\lambda}_{+}, \boldsymbol{\lambda}_{0}, \tau\right)=\left(\frac{1}{m} \mathbf{1}, \mathbf{0}, \tau\right)$ serves as a solution for any $\tau$. Hence $\left(\frac{1}{m} \mathbf{1}, \mathbf{0}\right)$ is an invariant pattern.

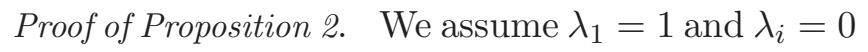
$(i=2, \ldots, K)$. Then we have

$$
\bar{v}=\sum_{i=1}^{m} \lambda_{i} v_{i}=v_{1}
$$

and, in turn, $v_{1}-\bar{v}=0$ thereby satisfying $\boldsymbol{F}_{+}(1$, $\mathbf{0}, \tau)=\mathbf{0}$. For $K-1$ places with no population, we have $\lambda_{i}=0$, thereby satisfying $\boldsymbol{F}_{0}(1, \mathbf{0}, \tau)=\mathbf{0}$. This shows that $\left(\boldsymbol{\lambda}_{+}, \boldsymbol{\lambda}_{0}, \tau\right)=(1, \mathbf{0}, \tau)$ serves as a solution for any $\tau$. Hence the monocenter at the place $i=1$ is an invariant pattern. Monocenters at other places can be shown as invariant patterns similarly.

Proof of Proposition 3 We consider two nodes $\left(n_{1}, n_{2}\right)$ and $\left(n_{1}^{\prime}, n_{2}^{\prime}\right)$. Then we have

$$
r^{3} p_{1}^{i} p_{2}^{j} \cdot\left(n_{1}, n_{2}\right) \equiv\left(-n_{1}-i,-n_{2}-j\right) \bmod n .
$$

Hence, any pair of $\left(n_{1}, n_{2}\right)$ and $\left(n_{1}^{\prime}, n_{2}^{\prime}\right)$ can be interchanged as

$$
\begin{aligned}
& g \cdot\left(n_{1}, n_{2}\right) \equiv\left(n_{1}^{\prime}, n_{2}^{\prime}\right), \\
& g \cdot\left(n_{1}^{\prime}, n_{2}^{\prime}\right) \equiv\left(n_{1}, n_{2}\right) \quad \bmod n
\end{aligned}
$$

by $g=r^{3} p_{1}^{i} p_{2}^{j}$ with $i=-n_{1}-n_{1}^{\prime}$ and $j=-n_{2}-n_{2}^{\prime}$. By choosing $G=\left\langle r^{3} p_{1}^{i} p_{2}^{j}\right\rangle$, we can show that a twin $(m=2)$ at any place is an invariant pattern by Lemma 1. The uniform state can be shown as an invariant pattern by extending the proof for the twins.

Proof of Proposition \& Since $G^{\prime}$ is a subgroup of $G$ with $|G|=\left|\left\langle r, s, p_{1}, p_{2}\right\rangle\right|=12 n^{2},\left|G^{\prime}\right|$ divides $12 n^{2}$ by Lagrange's theorem. The number $m$ of elements of an orbit divides $\left|G^{\prime}\right|$ Kochendörffer, 1970, Sec. 3.1.2]. Hence $12 n^{2}$ is divisible by $m$.

\section{Appendix B}

\section{Invariant Patterns for Lattice \\ Sizes $n=4,5,6$}

Invariant patterns for lattice sizes $n=4,5,6$ are presented. 


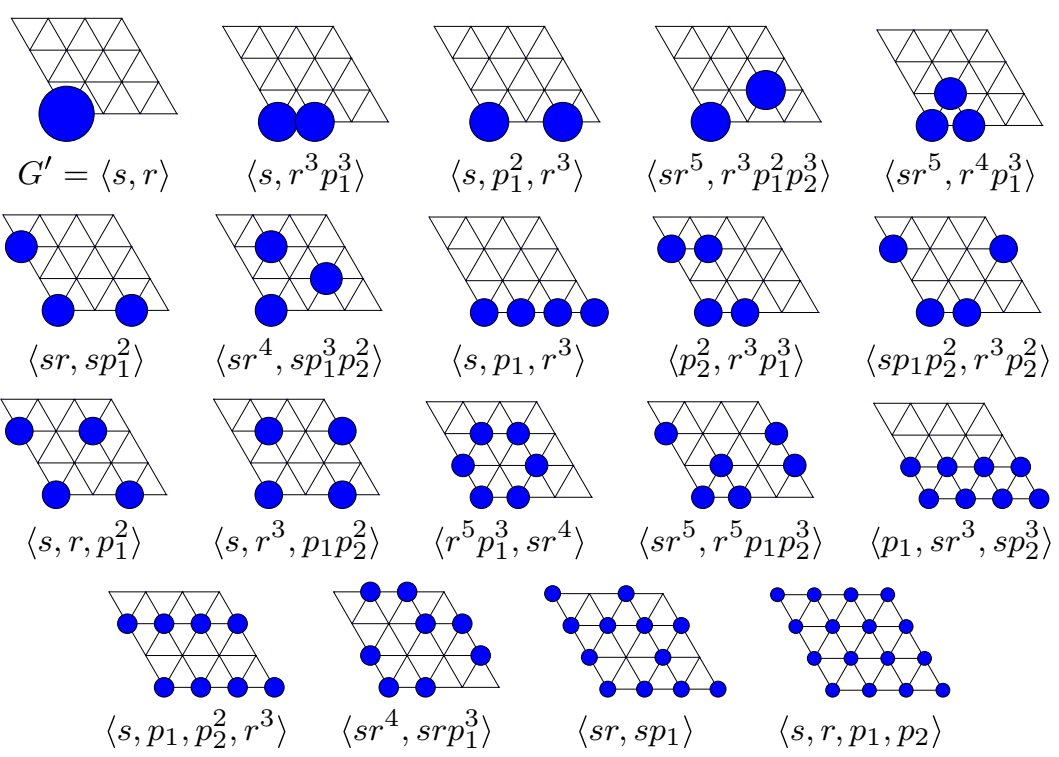

Fig. 11. Invariant patterns for $n=4$.
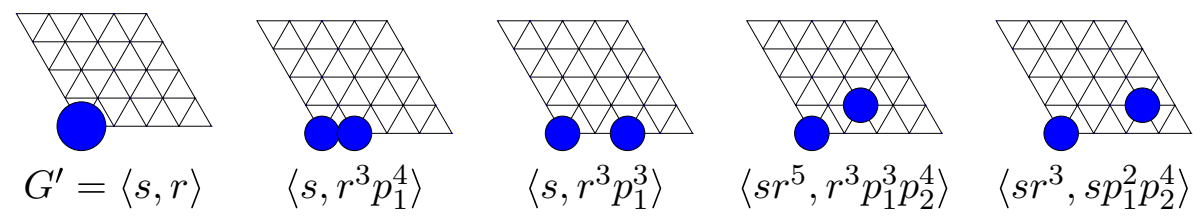

$\left\langle s, r^{3} p_{1}^{4}\right\rangle$

$\left\langle s, r^{3} p_{1}^{3}\right\rangle$

$\left\langle s r^{5}, r^{3} p_{1}^{3} p_{2}^{4}\right\rangle$

$\left\langle s r^{3}, s p_{1}^{2} p_{2}^{4}\right\rangle$
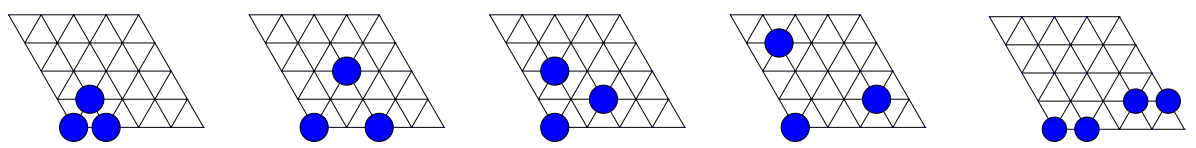

$\left\langle s r^{5}, r^{4} p_{1}^{4}\right\rangle$

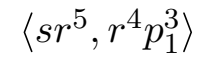

$\left\langle s r^{4}, s p_{1}^{4} p_{2}^{3}\right\rangle$

$\left\langle s r^{4}, s p_{1}^{2} p_{2}^{4}\right\rangle$

$\left\langle s p_{1}^{2} p_{2}^{4}, s r^{3} p_{1}^{4}\right\rangle$
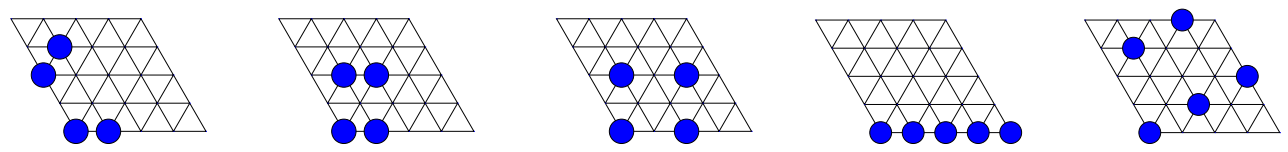

$\left\langle r^{3} p_{1}^{4}, s r^{5} p_{2}^{3}\right\rangle \quad\left\langle s p_{1}^{4} p_{2}^{3}, s r^{3} p_{1}^{4}\right\rangle$

$\left\langle s p_{1}^{4} p_{2}^{3}, s r^{3} p_{1}^{3}\right\rangle$

$\left\langle s, p_{1}, r^{3}\right\rangle$

$\left\langle s r^{5}, r^{3}, p_{1}^{2} p_{2}\right\rangle$
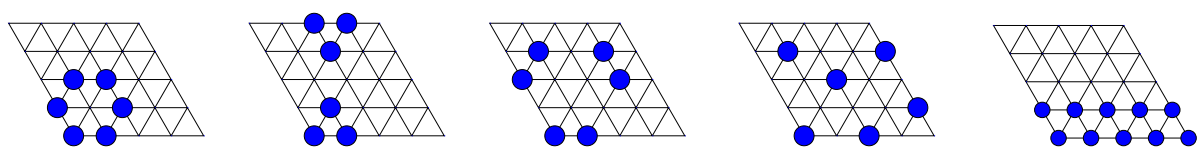

$\left\langle r^{5} p_{1}^{4}, s r^{4}\right\rangle \quad\left\langle s r^{5}, s p_{1}^{3} p_{2}\right\rangle$

$\left\langle s, r^{5} p_{2}^{3}\right\rangle$

$\left\langle s r^{5}, r^{5} p_{1} p_{2}^{4}\right\rangle \quad\left\langle p_{1}, s p_{2}^{4}, s r^{3}\right\rangle$
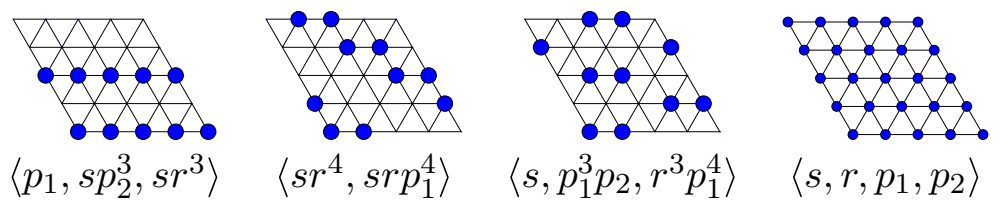

Fig. 12. Invariant patterns for $n=5$. 

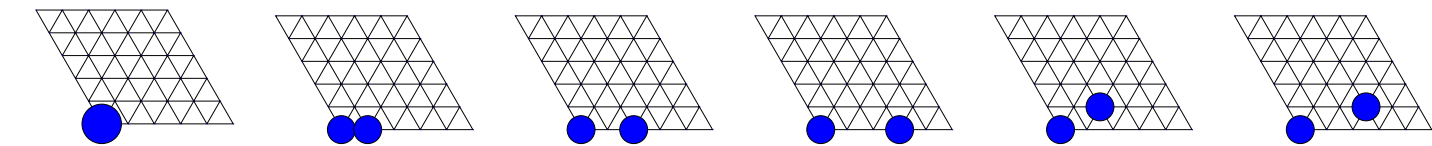

$G^{\prime}=\langle s, r\rangle$

$\left\langle s, r^{3} p_{1}^{5}\right\rangle$

$\left\langle s, r^{3} p_{1}^{4}\right\rangle$

$\left\langle s, r^{3}, p_{1}^{3}\right\rangle$

$\left\langle s r^{5}, r^{3} p_{1}^{4} p_{2}^{5}\right\rangle$

$\left\langle r^{3} p_{1}^{3} p_{2}^{5}\right\rangle$
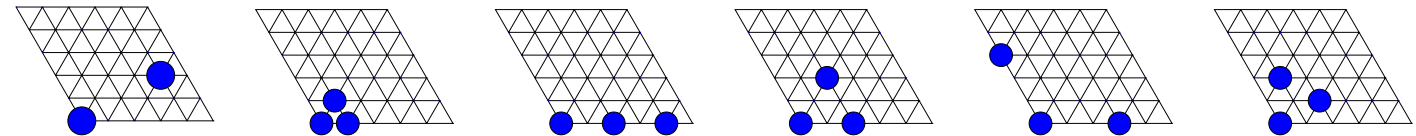

$\left\langle s r, s p_{1}^{2} p_{2}^{4}\right\rangle$

$\left\langle s r^{5}, r^{4} p_{1}^{5}\right\rangle$

$\left\langle s, p_{1}^{2}, r^{3}\right\rangle$

$\left\langle s r^{5}, r^{4} p_{1}^{4}\right\rangle$

$\left\langle s r, s p_{1}^{3}\right\rangle$

$\left\langle s r^{4}, s p_{1}^{5} p_{2}^{4}\right\rangle$
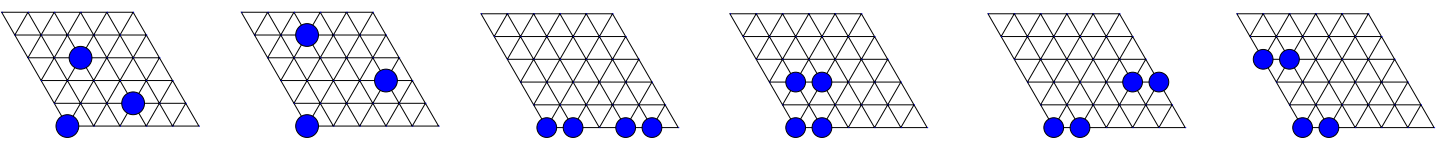

$\left\langle r^{4} p_{1}^{3} p_{2}^{5}\right\rangle$

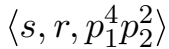

$\left\langle s, p_{1}^{3}, r^{3} p_{1}^{5}\right\rangle$

$\left\langle s p_{1}^{5} p_{2}^{4}, s r^{3} p_{1}^{5}\right\rangle$

$\left\langle s r^{3} p_{1}^{5}, s p_{1}^{2} p_{2}^{4}\right\rangle$

$\left\langle p_{2}^{3}, r^{3} p_{1}^{5}\right\rangle$
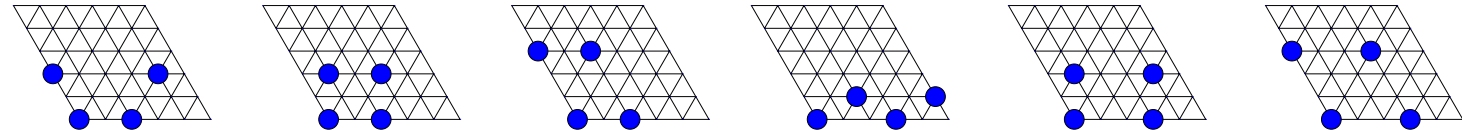

$\left\langle r^{3} p_{2}^{4}, s p_{1}^{2} p_{2}^{4}\right\rangle$

$\left\langle s p_{1}^{5} p_{2}^{4}, s r^{3} p_{1}^{4}\right\rangle$

$\left\langle p_{2}^{3}, r^{3} p_{1}^{4}\right\rangle$

$\left\langle s p_{1} p_{2}^{5}, s r^{3}\right\rangle$

$\left\langle p_{1}^{3}, s r^{3}, s p_{1}^{5} p_{2}^{4}\right\rangle$

$\left\langle s, r, p_{1}^{3}\right\rangle$
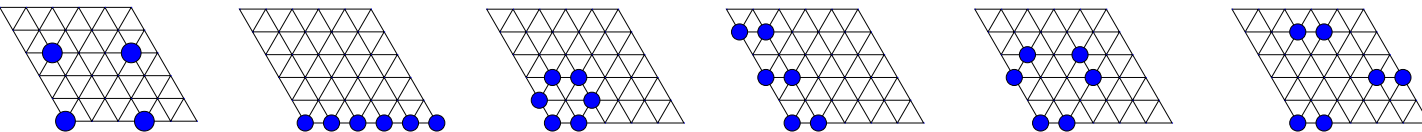

$\left\langle s, r^{3} p_{1}^{5} p_{2}^{3}\right\rangle$

$\left\langle s, p_{1}, r^{3}\right\rangle$

$\left\langle r^{5} p_{1}^{5}, s r^{4}\right\rangle$

$\left\langle p_{2}^{2}, r^{3} p_{1}^{5}\right\rangle$

$\left\langle s r^{5} p_{2}^{4}, s r^{3} p_{1}^{5}\right\rangle$

$\left\langle s, r^{3} p_{1}^{5}, p_{1}^{4} p_{2}^{2}\right\rangle$
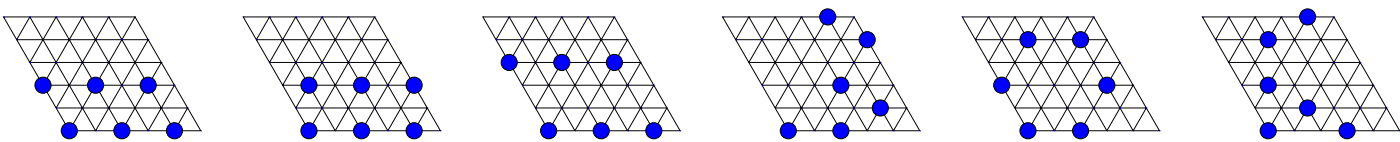

$\left\langle s p_{2}^{4}, s r^{3}\right\rangle$

$\left\langle s r^{3}, s p_{1} p_{2}^{4}\right\rangle$

$\left\langle p_{1}^{2}, r^{3}, p_{2}^{3}\right\rangle$

$\left\langle r p_{1}^{2} p_{2}^{5}\right\rangle$

$\left\langle s, r p_{1}^{4}\right\rangle$

$\left\langle s p_{1}^{3}, r^{2} p_{1}^{5} p_{2}^{4}\right\rangle$
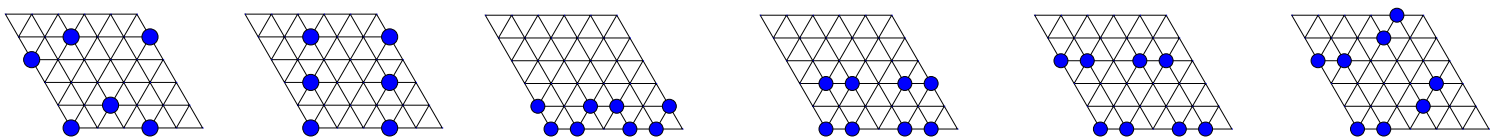

$\left\langle s r, s p_{1} p_{2}^{2}\right\rangle$

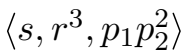

$\left\langle s p_{1} p_{2}^{5}, r^{3} p_{2}^{5}\right\rangle$

$\left\langle p_{1}^{3}, s p_{1}^{5} p_{2}^{4}, s r^{3} p_{1}^{5}\right\rangle$

$\left\langle s, p_{2}^{3}, r^{3} p_{1}^{5}\right\rangle$

$\left\langle r^{3} p_{1}^{5}, s r^{2} p_{1}^{2} p_{2}\right\rangle$
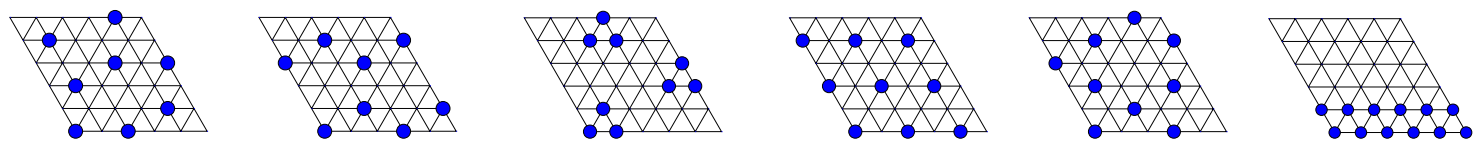

$\left\langle r^{3} p_{1}^{4}, s r p_{1}^{5} p_{2}^{2}\right\rangle$

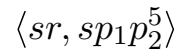

$\left\langle s r^{5}, r^{4} p_{1}^{5}, p_{1}^{4} p_{2}^{2}\right\rangle$

$\left\langle s, r, p_{1}^{2}\right\rangle$

$\left\langle s r^{4}, r p_{1}^{2} p_{2}\right\rangle$
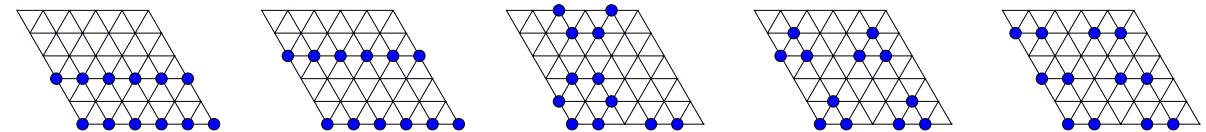

$\left\langle p_{1}, s p_{2}^{5}, s r^{3}\right\rangle$

$\left\langle p_{1}, s p_{2}^{4}, s r^{3}\right\rangle$

$\left\langle s, p_{1}, r^{3}, p_{2}^{3}\right\rangle$

$\left\langle s r^{5} p_{2}^{5}, s p_{1}^{3}\right\rangle$

$\left\langle s r^{5}, p_{1}^{3}, r^{4} p_{1}^{5}\right\rangle$

$\left\langle p_{2}^{2}, p_{1}^{3}, r^{3} p_{1}^{5}\right\rangle$
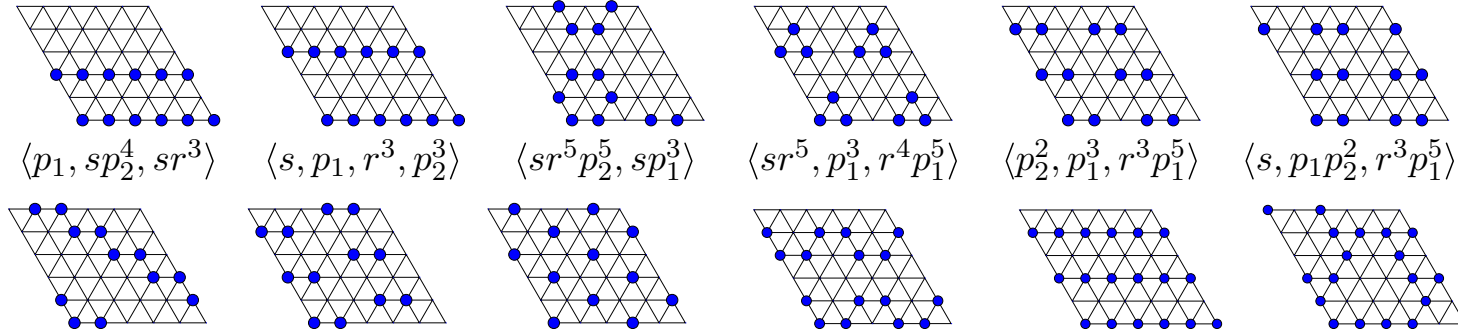

$\left\langle s, p_{1} p_{2}^{2}, r^{3} p_{1}^{5}\right\rangle$

$\left\langle s r^{4}, s r p_{1}^{5}\right\rangle$

$\left\langle r^{3} p_{1}^{5}, p_{1}^{3} p_{2}\right\rangle$

$\left\langle s, r, p_{1}^{5} p_{2}\right\rangle$

$\left\langle p_{2}^{3}, s p_{1} p_{2}^{5}, r^{3} p_{2}^{5}\right\rangle$

$\left\langle s, p_{1}, p_{2}^{2}, r^{3}\right\rangle$
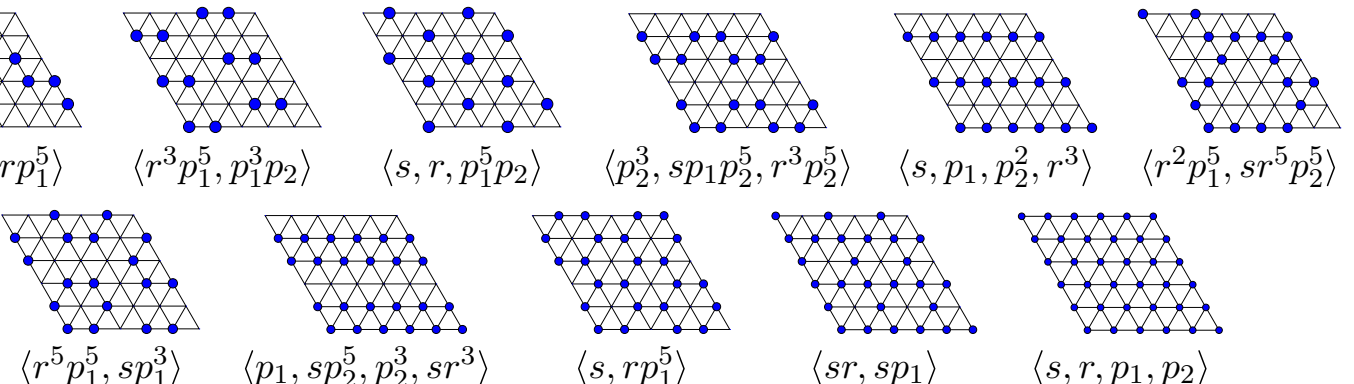

NOS

. .0 .0

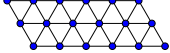

$\left\langle r^{5} p_{1}^{5}, s p_{1}^{3}\right\rangle \quad\left\langle p_{1}, s p_{2}^{5}, p_{2}^{3}, s r^{3}\right\rangle$

$\left\langle s, r p_{1}^{5}\right\rangle$

$\left\langle s r, s p_{1}\right\rangle$

$\left\langle s, r, p_{1}, p_{2}\right\rangle$

Fig. 13. Invariant patterns for $n=6$. 